\title{
Telecommunications Services and Multi-Unit Firms: Inside the Black-Box
}

\author{
ANNA CRETI * \\ Department of Economics and IEFE, Universita' Luigi Bocconi
}

\begin{abstract}
This paper analyzes telecommunications demand by firms, focusing on communication flows among production units. We consider first the case of two business unit that can play simultaneously or in a leader/follower situation regarding their communication decisions. In this context, we define the network effect as the advantage a production unit obtains by using an input whose costs are shared by another user. The model is then generalized, taking into account indirect links among production units. The theoretical framework we suggest explains business telecommunications usage by multi-unit firms, and sheds light on the relationship between firms' internal organisation and communication flows.
\end{abstract}

\section{Introduction}

Business telecommunications demand is one of the areas most in need of attention in the evaluation of how the telecommunication services provision is changing. However, despite the interest in understanding how firms use telecommunication value added services (that is, virtual private networks, integrated services with data and voice transmission, leased lines, mobile communication) and a growing number of business tariff options, both theoretical and econometric analyses of the basic firms' telecommunications needs are difficult to find.

In order to study telecommunications business demand, some authors have attempted to focus on business telecommunications demand in aggregate models (Perez-Amaral et al., 1995 and Curien-Gensollen, 1989). The use of aggregate data is useful to understand the general trend of business telecommunication demand, but "firms vary a great deal from one to another in terms of their productive structure, and this requires, when possible, a disaggregation in the study of the telecommunication demand" (Perez-Amaral et al., 1995 page 45). Nevertheless, micro-econometric analyses of telecommunications usage by firms are even more difficult to find than aggregate analyses: to our knowledge, only one study analyzes firms' data (Griffin, 1989), focusing on substitution relationships among business

\footnotetext{
* Università L. Bocconi and IEFE, Via Sarfatti 25, 20136 Milan, Italy; tel 003902583638 12, fax 025836 38 90. Email: anna.creti@unibocconi.it An earlier version of this paper was entitled "Communication flows and firms' organization: the impact of the network externality effect on the production function". I am very grateful to Alain Bousquet, Marc Ivaldi, Said Souam, Patrick Rey, Michel Wolkowicz, and an anonymous referee for their useful suggestions. All errors remain mine.
} 
intercity telecommunications service, treated as multiple goods in the firms' production function.

The difficulty of finding micro-econometric studies on business telecommunication demand is essentially due to two important factors: the first one is technical - panel data or simple cross-section telecommunications usage by firms data is scarce; the second one is more conceptual - once an individual piece of information is obtained, it is not clear how to model firms' telecommunications demand. Although the type of access to telecommunication services (local or medium-long distance, single or multiple lines, leased lines) is an important determinant of business demand, the main focus of business telecommunications demand is how to model usage, since no business can be without basic telephone service. However, "attempts to model business telecommunications demand in terms of generic needs of a generic firm, or analogously to the residential customers, are simply not very useful" (Taylor, 1994, page 120).

In an empirical study that analyzes telecommunications usage on cross-section data (Bousquet-Creti-Wolkowitcz, 2000), we have experienced the lack of a well adapted approach for modelling business communication services. We used a very complete dataset, the "Base Marketing Enterprises-France Télécom", which provides information on a representative sample of France Télécom business customers having a budget for telecommunication services of more than 45,000 Euros by year and positive local, national and international traffic. We treated local, national and international calls as multiple goods that are inputs in the firms' production function, together with labor, considered as a fixed short-term input. Based on cost minimization and duality theory, a translog cost function was estimated, allowing for imperfect substitution among local, national and long distance traffic.

In Bousquet-Creti-Wolkowitcz, we show that the estimation of the cost function exhibited a good fit $\left(\mathrm{R}^{2}=0.58\right)$ and discuss two important results. First, structural characteristics, such as firms' sector, mono or multi-location, geographic plant dispersion, tend to be quite important in determining business telecommunication demand. Compared to these determinants, telecommunications price appears relatively less important (estimated price elasticities are quite low in absolute value). Second, some omitted variables not observed in our database, mainly the information about internal communication networks linking different plants and the technical characteristics of telecommunications equipment at the plant level, have lowered the estimation goodness of fit, especially for the cost share of national traffic $\left(\mathrm{R}^{2}=0.15\right)$.

If the impact of firms' structural characteristics is quite intuitive and widely discussed (Taylor, 1994; Delmastro, 2004), then the second effect, namely the impact of firms' internal communication networks on business telecommunications demand, deserves particular attention (Marinucci and Perez-Amaral, 2005). Internal communication networks allow firms to create dedicated links between all, or also just a portion of their plants, benefiting from particular tariffs. Actually, beside these more usual telecommunication services, the most sophisticated type of internal networks is "intranet", that is, internet services among different business units belonging to a firm or an administration. Management reviews often describe case studies of firms adopting intensive internal communication strategies (attractively defined e-strategies), and information technology consultants stress the importance of the network infrastructure (hardware) and usage (software). 
In general, virtual private networks are adopted by medium and large firms that are in the categories where our estimation for national telecommunications flows seems to be the most imprecise. But how can we model communication networks among different plants? To our knowledge, the complex interaction between internal communication networks and business telecommunications demand has never been analytically studied. While in the standard literature on telecommunications residential demand the idea of access network externalities is very well characterized (the value of a network increases each time an additional user joins), our approach is a new one, in which we underline that network externalities may influence usage of telecommunication services and therefore the production function, acting as technical production externalities. The production function depends on labor and telecommunications services equipment linking different nodes. The magnitude of the network externality effect depends both on network size and on the intensity of interaction among users (the externality parameters). We discuss the implications of this strategic interaction, an aspect that has been neglected thus far by the existing literature on telecommunication and IT usage by firms.

Units optimally allocate the resources between labor and telecommunications infrastructure, and subsequently optimize usage, that is, number (or duration) of calls. Several aspects are at stake in our model. In the first part of the paper, we consider a 2nodes model, by focusing on the outcomes of the simultaneous versus the sequential setting (Section 2). In the simultaneous setting, both units consider only that the volume of information they obtain is a function of their calls as well as the other unit's incoming contacts. As a preliminary result, we show that at the equilibrium that guarantees a unit's cost minimization, the marginal productivity of the input telecommunication increases with respect to the case where network externalities are not taken into account. Moreover, when one unit acts as a leader in the communication usage, that is, she minimizes her production costs by considering the networking function of the other unit, it can lower her demand of telecommunications infrastructure with respect to the simultaneous setting. The simultaneous setting is probably the most common one, whereas the sequential scenario assumes that one unit is the "communication leader" as her role is to process information and then to take advantage of reports made by other units.

In the second part of the paper, we analyze the impact of more complex network configurations on telecommunication infrastructure and usage (Section 3). To this end, we slightly modify the model's structure by making the assumption that the volume of information one unit obtains is a function of her own calls and of the total volume of information held by the correspondents. The proxy used to measure the total volume of information is the network infrastructure. Clearly, each unit approximate a fraction of the total volume of information held by other units. The extent of this beneficial effect is measured by an externality parameter. Our main result is that direct links tend to increase the need for telecommunications equipment, because the value of each unit of information is maximum for direct transfers. However, indirect links tend to counterbalance this effect because each node can benefit from using the network as a common resource, whose costs are shared with other subscribers. We show that an increase in externality parameters increases the value of communicating with the nodes with which there are direct links, but also gives incentive to take advantage of the global network externalities (generated by indirect links) and thus to lower costly usage. We explain that in a 3-nodes network, a unit $i$ which is connected with two units $j$ and $k$ will derive a different demand for telecommunications infrastructure and number of calls depending on whether units $j$ and $\mathrm{k}$ 
are themselves directly connected. This result comes from the fact that if these units $j$ and $k$ are directly connected then unit $i$ will benefit from a supplementary network externality, which will encourage it to lower its own usage. Therefore, the usage of a specific node in the structure is maximum when it has only direct links with the other nodes.

We identify two major determinants of telecommunication usage by multi-unit firms:

- the increase of marginal productivity of telecommunication infrastructure: this is the direct or productivity effect;

- the substitution with telecommunication usage (that is, the number of calls) by other units: this is the network or free-rider effect.

These two effects combine in various ways depending on the hierarchical role of the units in the network, the structure of the contacts, the degree at which a unit appropriates the total volume of information of the other network users. We conclude the paper discussing some applications of our model (Section 4) in the econometrics of business telecommunication demand, and more broadly, in the context of the literature on firms' internal organization and information processing. All the proofs are relegated to the Appendix.

\section{Production in presence of network externalities}

In order to analyze the effect of network externalities on the production function, we use a mix of different approaches: the traditional theory of residential telephone demand (Artle and Averous, 1973; Squire,1973; Rohlfs, 1974 and more recently, Taylor, 1994; and Bousquet and Ivaldi, 1997), as well as the fundamental models on network externalities (see for example, Katz and Shapiro, 1985, 1986; Economides, 1991; and Liebowitz and Margolis, 1994). In general, these papers analyze the role played by network effects for the adoption of telecommunication services, which lead to diffusion phenomena including critical mass, lock-in, path dependency, and inefficiency, except for Bousquet and Ivaldi, who point out the importance of usage network externalities. Our simple framework is different as telecommunications services are an input of the production function and interaction between different production units through telecommunication services creates the rationale for network effects. Therefore, we focus on the impact of the network effect on telecommunication demand, both on infrastructure or equipment (number of switches and telephones, but also computers, if we think more specifically to IT services) and on usage (number of calls or minutes of communication). Regarding this specific aspect, our analysis is similar to the problem of cost allocation of a resource that is shared among several departments (see for example, Gal-Or, 1993), with the additional aspect that the efficiency of the common resource, that is the telecommunication network, may increase as the extent of utilization by other firm's units increases.

We consider first two symmetric production units. The central manager of the firm has a specific production target for these two units and provides them with access to a leased line or to a virtual private network. The optimal mix of input, telecommunication services and labor, is totally decentralized to the unit level.

These assumptions are quite realistic: one can think of a bi-divisional corporation, where the CEO decides which car model each unit will produce and chooses the type of 
access to telecommunication facilities (local or medium-long distance, single or multiple lines, leased lines) or the type of services (internet, electronic mail). The infrastructure purchase as well as the usage intensity is therefore delegated to single divisions ${ }^{1}$.

Let us assume that unit $I$ 's input, labor $L_{i}$ and telecommunication service infrastructure $N_{i}$, are combined by a standard Cobb-Douglas production function with constant return to scale ${ }^{2}$ :

$$
Y_{i}=L_{i}^{\beta} N_{i}^{1-\beta} \quad i=1,2 \quad 0<\beta<1 .
$$

The telecommunication equipment $N$ has to be set up taking into account links between units through a communication network, from which the following networking function of unit 1 (unit 2 being perfectly symmetric):

$$
N_{1}=\bar{N}_{1}+\varepsilon_{21} \bar{N}_{2} 1>\varepsilon_{21}>0 .
$$

In the above networking function, $\bar{N}_{1}$ represents usage, that is, number of outgoing calls from unit 1 to unit 2, and $\bar{N}_{2}$ is interpreted as the number of incoming calls from unit 2 , whose efficiency is represented by the parameter $\varepsilon_{21}$.

Here, we obviously refer only to contacts that contain the relevant information for the production process, under the hypothesis that $\bar{N}_{1}$ and $\bar{N}_{2}$ are imperfect substitutes ${ }^{3}$. Moreover, we assume $\varepsilon_{21}<1$, as contacts generated by the interested unit have a higher probability of being more relevant for that unit than the calls received. This formulation also allows us to point out the concept of call externalities or the benefit of being called.

Equations (1) and (2) together show the impact of the network of users in the production function. On one side, the network size or the number of users has a positive impact on $N$, which also represents a measurable proxy for the volume of information the user can gather, as in the traditional theory on telephone demand. A larger $N$ also increases production. On the other side, as $\bar{N}_{1}$ and $\bar{N}_{2}$ are substitutes, the units can substitute their direct calls with those of the others belonging to the network in order to get specific information.

As regards the cost function, the unit costs of labor are denoted by $c_{L}$. We assume that units choose among different kinds of telecom equipment, having different quality and capacity and will then allocate their resources proportionally to $N$ and $\bar{N}$. Therefore, the costs of contacts are the sum of costs related to the purchase of the necessary equipment $c_{n} N_{i}$, and those related to usage, as a function of the number of calls, $c_{\bar{N}} \bar{N}_{i}$ (with $c_{\bar{N}}<c_{n}$ ). The networking cost function is:

$$
C\left(N_{i}, \bar{N}_{i}\right)=c_{n} N_{i}+c_{\bar{N}} \bar{N}_{i} i=1,2 \quad i \neq j .
$$

In the following section, we are going to analyze two possible scenarios. In the first case, each of the two units minimizes its cost function under the constraint given by the networking function (equation 2). In the second case, we assume that unit 1 acts as a leader

\footnotetext{
${ }^{1}$ Evidence of this kind business practices in organising the provision of telecommunication services is given by Odlyzko (2000).

2 The hypothesis of constant returns to scale is not crucial, but simplifies the analytical results.

${ }^{3}$ Notice that we focus on the case of positive network externalities only. Congestion effects could be analyzed using negative externalities parameters.
} 
in the telecommunication setting, and minimizes its costs taking into account that in equation (2), the incoming calls are calculated by equation (3b); unit 2 only considers the networking function as given by $N_{2}=\bar{N}_{2}+\varepsilon_{12} \bar{N}_{1}$. We then discuss the difference in the equilibria obtained in the simultaneous setting versus the sequential one.

\subsection{Optimal telecommunication infrastructure and calling plan}

As the production units are price-taking in the output market, we analyze optimal resource allocation among labor and telecommunication services, taking into account the interdependence between $\bar{N}_{1}$ and $\bar{N}_{2}$.

\subsubsection{Simultaneous setting}

When minimizing its production costs (the sum of labor costs and networking costs), firm $i$ considers as given the output level and the number of calls of its correspondent. The cost minimization program is:

$$
\begin{aligned}
& \underset{L_{1}, N_{1}}{\operatorname{Min}} C_{t o t}=c_{L} L_{1}+c_{n} N_{1}+c_{\bar{N}} \bar{N}_{1} \\
& \text { s.t. } \quad Y^{*}-L_{1}^{\beta} N_{1}^{1-\beta}=0 \\
& \bar{N}_{1}+\varepsilon_{21} \bar{N}_{2}=N_{1} .
\end{aligned}
$$

Using equation (2), the constraint resulting from the networking function can be solved for $\bar{N}_{1}$ and substituted into the objective function as follows:

$$
\begin{aligned}
& \underset{L_{1}, N_{1}}{\operatorname{Min}} C_{\text {tot }}=c_{L} L_{1}+\left(c_{n}+c_{\bar{N}}\right) N_{1}-\varepsilon_{i j} c_{\bar{N}} \bar{N}_{2} \\
& \text { s.t. } \quad Y^{*}-L_{1}^{\beta} N_{1}^{1-\beta}=0 .
\end{aligned}
$$

The marginal cost of the network infrastructure are $\partial C\left(N_{i}\right) / \partial N_{i}=c_{n}+c_{\bar{N}}$, while $\bar{N}_{2}$ is a subsidy. Therefore, on one side, a production unit has incentives to increase her calls to benefit of the communication network and increase production but on the other side, she can also free ride and let the other production unit call, decreasing her own telecommunication costs.

The first order conditions (FOC) give the demand function for telecommunication services that guarantees costs minimization ${ }^{4}$ :

$$
N_{1}^{*}=Y_{1}^{*}\left(\frac{1-\beta}{\beta} \frac{c_{L}}{C^{\prime}\left(N_{1}\right)}\right)^{\beta},
$$

where $C\left(N_{1}\right)=c_{n}+c_{\bar{N}}$.

Combining equation (4) with the networking function, we obtain:

$$
\bar{N}_{1}=N^{*}{ }_{1}-\varepsilon_{21} \bar{N}_{2} \text {. }
$$

Symmetrically, for unit 2 we have:

\footnotetext{
${ }^{4}$ The second order conditions are always satisfied, because the objective function is linear and the constraint functions are concave or linear (respectively for the production and the networking functions).
} 


$$
\bar{N}_{2}=N^{*}{ }_{2}-\varepsilon_{12} \bar{N}_{1} \text {. }
$$

Given the interdependence between $\bar{N}_{1}$ and $\bar{N}_{2}$, equations (5) and (6) can be interpreted and thus solved as a system of reaction functions. The equilibrium is as follows:

$$
\begin{aligned}
& \bar{N}_{1}^{*}=\frac{1}{1-\varepsilon_{21} \varepsilon_{12}}\left(N_{1}^{*}-\varepsilon_{21} N_{2}^{*}\right), \\
& \bar{N}_{2}^{*}=\frac{1}{1-\varepsilon_{21} \varepsilon_{12}}\left(N_{2}^{*}-\varepsilon_{12} N_{1}^{*}\right) .
\end{aligned}
$$

Notice that under the assumptions on the externality parameters, we have $0<1-\varepsilon_{21} \varepsilon_{12}<1$. At the equilibrium, the number of calls of unit $i$ is finally determined by the choice of $N_{i}$ and $N_{j}$, thus showing the interdependence between users. In fact, the difference in the allocation of the traditional model of production factors, given a certain level of output, is that the optimal number of calls depends not only on the choices of unit 1 , but also on what unit 2 decides (and vice versa). Moreover, unit $i$ 's usage increases as long as the efficiency of its calls toward unit $j$ increases (that is, when $\varepsilon_{i j}$ increases), and vice versa, if the benefit from calls received by unit $\mathrm{j} \varepsilon_{j i}$ increases, $\bar{N}_{i}^{* N}$ decreases.

The result in the presence of a leadership in the communication decision-making is analyzed in the next paragraph, where we will explore an additional feature of the network externality effect: the non-compensation of the benefits obtained through the telecommunications' network.

\subsubsection{Sequential setting}

We now suppose that unit 1 is the leader and unit 2 the follower: this can correspond to the behavior of a functional-oriented organization (the marketing-led M-form), where the marketing unit decides production based on her own information, but also using a "report" from production, as argued in Carter (1996). While the follower's problem is still to minimize the production costs for a target output, taking the decision of unit 1's as given, the leader (henceforth $l$ ) achieves cost minimization taking into account the networking function of unit 2:

$$
N_{1}^{l}=\varepsilon_{21} \bar{N}_{1}^{l}+\varepsilon_{12}\left(\frac{N_{2}-\varepsilon_{12} N_{1}^{l}}{1-\varepsilon_{21} \varepsilon_{12}}\right)
$$

or

$$
\bar{N}_{1}^{l}=\frac{N_{1}^{l}}{1-\varepsilon_{21} \varepsilon_{12}}-\frac{\varepsilon_{12} N_{2}}{1-\varepsilon_{21} \varepsilon_{12}} .
$$

Therefore, the network cost function of the communication leader is as follows:

$$
C\left(N_{1}^{l}\right)=\left(c_{n}+\frac{c_{\bar{N}}}{1-\varepsilon_{12} \varepsilon_{21}}\right) N-c_{\bar{N}} \frac{\varepsilon_{12} N_{2}}{1-\varepsilon_{21} \varepsilon_{12}} .
$$

Unit 1's FOCs for cost minimization imply:

$$
N_{1}^{l}=\frac{c_{L}}{\left(c_{n}+\frac{c_{\bar{N}}}{1-\varepsilon_{12} \varepsilon_{21}}\right)} \frac{1-\beta}{\beta} L .
$$


The demand for the infrastructure is therefore:

$$
N_{1}^{l}=\frac{Y_{1}^{*}}{1-\varepsilon_{21} \varepsilon_{12}}\left(\frac{1-\beta}{\beta} \frac{c_{L}}{C^{\prime}\left(N_{1}^{l}\right)}\right)^{\beta},
$$

where:

$$
C^{\prime}\left(N_{1}^{l}\right)=c_{n}+c_{\bar{N}}\left(\frac{1}{1-\varepsilon_{21} \varepsilon_{12}}\right) \text {. }
$$

Finally, the equation for the number of contacts of the leader is obtained by using the networking function:

$$
\bar{N}_{1}^{* l}=\frac{1}{1-\varepsilon_{21} \varepsilon_{12}}\left(N_{1}^{l}-\varepsilon_{21} N_{2}^{*}\right)
$$

where:

$$
N_{2}^{*}=Y_{2} *\left(\frac{1-\beta}{\beta} \frac{c_{L}}{C^{\prime}\left(N_{2}\right)}\right)^{\beta} .
$$

Comparing the solutions to the simultaneous and the sequential setting, we obtain the following results:

\section{Proposition 1}

Unit 1 's demand for telecommunications infrastructure is lower in the sequential setting than in the simultaneous setting.

\section{Corollary 1}

The number of calls generated by the leader (unit 1) in the sequential setting is lower than the number of contacts he generates in a simultaneous market situation, while the follower (unit 2) increases the number of calls with respect to the simultaneous setting. Moreover, at equilibrium, the leader's technical rate of substitution between labor and communication infrastructure is higher than in the simultaneous setting.

Proof: See the Appendix.

The intuitions behind these results are very simple. The temporal asymmetry allows unit 1 to increase the marginal productivity of the telecommunication infrastructure. That effect (similar to an increase in outgoing calls efficiency in the simultaneous game) resembles the hicksian technological progress, which allows unit 1 to achieve the same production level with lower telecommunication services, but is related to the noncompensation of an input whose costs are shared with another user: this is the network of free-riding effect. Indeed, not only the advantage unit 1 gains in the production function is due to the interdependence between the users, but also the non-compensation of the network effect here takes the specific form of a productivity effect not paid by the firm. In fact, the sequential game outcome can be explained as a clockwise rotation in the leader's reaction function, due to the higher network effect. The greater this network effect (that is, $\left.\varepsilon_{12} \varepsilon_{21}\right)$, the higher the shift, hence the lower the sequential solutions of unit 1 , because the network marginal costs increase, thus the demand for $N$ decreases. In our model, $\bar{N}_{2}$ is a substitute of $\bar{N}_{1}$, but its increase has a positive effect on the unit's objective function 
$\left(\partial C_{1} / \partial \bar{N}_{2}<0\right)$. This means that when unit 1 becomes the leader in the calling plan, she free-rides, forcing unit 2 to increase its number of calls. ${ }^{5}$ In fact, empirical studies show that more incoming calls relative to outgoing calls are observed in leader units, such as firms' headquarters (Bousquet and Joram, 1994).

Moreover, the network effect also leads to the increase of the rate of substitution between labor and communication infrastructure in the sequential game with respect to the simultaneous one.

Finally, one may think that, given the posited externalities in the communication process between the two units, a centralized decision would be better. In fact, straightforward calculations would show that, jointly minimizing the production costs of the two units, the presence of externalities in a market implies a lower amount of demand compared to the optimum. Therefore, the fact that the CEO only decides the type of access for communication network between the two units is inefficient.

\section{A generalisation with different network configurations}

We now extend our model, not only to take into account more than two units, but mainly to show that the entire structure of the communication network affects the demand for each given link. Indeed, comparing optimal choice of telecommunication infrastructure and calling plans for different network configurations, we point out that the structure of the network determines its usage.

To start as simply as possible, we focus on the case of three units, although most insights can be generalized for $n$ units. In our network structure, units can be linked together, directly or indirectly, in different ways. Focusing on the pivotal position of unit 1, three different kinds of linkage can be considered (Figure 1):

- full connectivity, where all units are directly connected to each other;

- $\quad$ serial connectivity, where unit 1 is directly connected with unit 2 , and unit 2 with unit 3, but no direct linkage between 1 and 3 exists;

- partial connectivity, where unit 1 is directly linked to both units 2 and 3 but there is no direct link between those two units.

How can these structures affect the units' networking functions? Each time unit $i$ contacts other users, she benefits from a fraction of their communication total architecture and gathers $\sum_{j} E_{i j} N_{j}$, where the total amount of information obtained from contacting other units $j$ is weighted by the exogenous parameters $E_{i j}<1$, meaning the externality effect or the quality/benefit of exchange unit $i$-unit $j$.

\footnotetext{
${ }^{5}$ This equilibrium can be dynamically unstable, because the telecom investment is neither a real barrier to entry, (the model neglect imperfect competition) nor irreversible (only one network is available). System compatibility and quasi-irreversibility of investment in specific touch-typing skills become very important in the transition to another network. We could explain the dynamic value of a specific investment in the telecommunication input by analyzing the technological choice among two incompatible networks. This is beyond the scope of this paper and is left for further research.
} 


\section{Full connectivity}

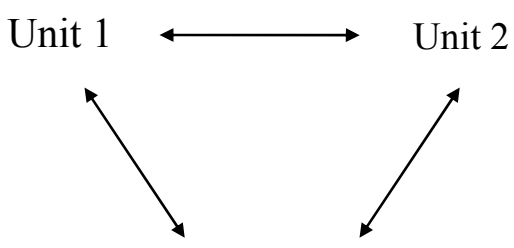

Unit 3

\section{Serial connectivity}

Unit $1 \longmapsto$ Unit 2

\section{Partial connectivity}

Unit 3

Unit $1 \longleftrightarrow$ Unit 2

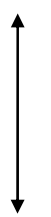

Unit 3

\section{Figure 1: Network configurations}

For simplicity, we assume that $N_{i}$ is a linear function ${ }^{6}$ of $\bar{N}_{i}$ and of $\sum_{j} E_{i j} N_{j}$. Because of indirect links, $N_{j}$ will depend on $\bar{N}_{j}$, and on the contacts between $j$ and the other units linked to $j$, since:

$$
N_{j}=\bar{N}_{j}+\sum_{k} E_{j k} N_{k}
$$

Taking into account the direct as well as indirect links, the networking function for unit $i$ becomes:

$$
N_{i}=\bar{N}_{i}+\sum_{j} E_{i j}\left(\bar{N}_{j}+\sum_{k} E_{j k} N_{k}\right)
$$

\footnotetext{
${ }^{6}$ This corresponds to the usual formulation of spillovers in the R\&D literature (see for example, De Bondt, 1996). However, R\&D models totally ignore the idea of indirect links among units, which is the main characteristic of our modelling of telecommunication services.
} 
Following this logic, if unit $k$ has other indirect links, they will be integrated in the unit $i$ 's networking function. This process will stop when every indirect link of the network has been considered". In this mechanism of "diffusion of knowledge", in addition to $E_{i j}$, the externality effect of the exchange unit $i$ - unit $j$, other externality parameters appear (all less than 1):

$E_{i j} E_{j i}=E_{i}^{j}$ as the benefit from the two-way exchange unit $i$ - unit $j$;

$E_{i k} E_{k j}=E_{i j}^{k}$ as the benefit from the indirect exchange unit $i$ and $j$, through unit

$k$;

$E_{i j} E_{j k} E_{k i}=E_{i i}^{j k}$ as the benefit from the circuit among unit $i, j, k$.

Finally, the networking function of each unit is the solution of a system of linear equations where $\bar{N}_{i} \quad i=1,2,3$ are constant. For example, in the case of serial connectivity:

$$
\begin{aligned}
& N_{1}=\bar{N}_{1}+E_{12} N_{2} \\
& N_{2}=\bar{N}_{2}+E_{21} N_{1}+E_{23} N_{3} \\
& N_{3}=\bar{N}_{3}+E_{32} N_{2} .
\end{aligned}
$$

Solving the system, it results that unit $i$ 's networking function $N_{i}$, taking into account the entire structure of the network, is in a linear function of her own telecommunication usage $\left(\bar{N}_{i}\right)$, the telecommunication usage of units contacted directly $\left(\bar{N}_{j}\right)$, and indirectly through $j\left(\bar{N}_{k}\right)$, and also of the various externality parameters. Hence, $\bar{N}_{j}$ and $\bar{N}_{k}$ are substitutes of $\bar{N}_{i}$, but their degree of substitutability depends, in a complex way, not only on the externality effects generated by direct contacts between unit $i$ and unit $j$, but also on those benefits arising from the indirect links unit $i$ - unit $k$, through unit $j$.

To better understand how network structure affects telecommunication infrastructure demand and usage, we assume that the externalities are isotropic, meaning that a unique parameter is associated with each interaction among firms: $E_{i j}=E_{j i}=E<1$. This hypothesis also implies $E_{i j} E_{j k}=E_{i k}^{j}=E^{2}$ and $E_{i j} E_{j k} E_{k i}=E_{i i}^{j k}=E^{3}$.

To simplify the presentation, we write the networking functions system in the matrix form:

$$
N=\bar{N}+\widetilde{E} N
$$

where $\boldsymbol{N}$ is the vector $(1 \times 3)$ of the telecommunication infrastructure variables, $\overline{\boldsymbol{N}}$ is the vector (1x3) of the telecommunication usage, and $\widetilde{\boldsymbol{E}}$ is the matrix (3x3) of the efficiency

\footnotetext{
${ }^{7}$ In a topological approach, this process would be the evaluation of the oriented graph obtained in the serial, partial or full connectivity structure, where $\bar{N}_{i}$ is the value associated with the node $i$ and $E_{i j} N_{j}$ is the value of the arc originating from the node $i$ toward the node $j$. Furthermore, our networking function has a meaning very similar to the "value function" of a network as used in some papers in the cooperative game theory (Jackson-Wolinsky, 1996; and Dutta-Mutuswami, 1997), to indicate the output of the agents when they are organized according to a particular graph.
} 
parameter $E$ describing communication flows among the users. Under all configurations, the principal diagonal of $\widetilde{\boldsymbol{E}}$ is zero, because obviously units do not get new information by talking to themselves. One can embed full, serial and partial connectivity within this general framework, simply by setting some of the elements of $\widetilde{\boldsymbol{E}}$ equal to zero, when the direct link between two units does not exist.

The solution for $\boldsymbol{N}$ in terms of $\overline{\boldsymbol{N}}$, that is, the networking functions, is given by $\boldsymbol{N}=(\boldsymbol{I}-\widetilde{\boldsymbol{E}})^{-1} \overline{\boldsymbol{N}}$. Therefore, we calculate the parameters value under which $(\boldsymbol{I}-\widetilde{\boldsymbol{E}})^{-1}$ exists (that is, the determinant is different from zero) and is positive definite (the determinants of all the principal submatrices are strictly positive $)^{8}$, in order to get positive vectors of choice variable $N$. Notice that if the matrix $(\boldsymbol{I}-\widetilde{\boldsymbol{E}})^{-1}$ was negative definite, congestion, that is, negative externality effects arise. Therefore, to ensure positive network effects we may end up with quite low values of the externality parameters. In fact, with fixed capacity of the line, the higher the number of direct links, the more likely is congestion.

Once we have obtained the networking functions in the serial/partial/full connectivity cases, we must solve the cost minimization problem. To fix ideas, we will analyze the impact of different network configurations on unit 1's costs minimization. As in section 2, unit 1 minimizes her production costs given the output level $Y^{*}$ and the networking functions. Under the different network structures, unit 1 decides her demand for inputs, and then, in particular, on $N_{1}$ and $\bar{N}_{1}$, taking as given $\bar{N}_{2}$ and $\bar{N}_{3}$, and the externality parameters. All units are symmetric and achieve Nash equilibrium in their decision variables.

In paragraph 3.1, we derive the network connectivity and cost functions associated with each network structure and we solve unit 1's cost minimization problem under different network configurations, while in paragraph 3.2, we compare the different reaction functions and the Nash equilibrium associated with the full/serial/partial connectivity cases.

\subsection{Cost minimization under different network configurations}

We start from the symmetric situation where each unit is linked to all the other users: full connectivity. Once we have obtained the network connectivity and the cost functions, we minimize production costs. Discussion of serial and partial connectivity follows.

\subsubsection{Full connectivity}

In the full connectivity configuration, all the units are symmetric. Networking functions are obtained from the following system of linear equations in the matrix form:

$$
\left[\begin{array}{l}
N_{1} \\
N_{2} \\
N_{3}
\end{array}\right]=\left[\begin{array}{l}
\bar{N}_{1} \\
\bar{N}_{2} \\
\bar{N}_{3}
\end{array}\right]+\left[\begin{array}{lll}
0 & E & E \\
E & 0 & E \\
E & E & 0
\end{array}\right]\left[\begin{array}{c}
N_{1} \\
N_{2} \\
N_{3}
\end{array}\right] .
$$

In order to solve the system with positive $N_{i} i=1,2,3$ we consider values of $E<0.5$. The networking function of unit 1 in the full connectivity case (henceforth $F$ ) is as follows:

\footnotetext{
${ }^{8}$ For these general properties of matrices, see Basil-Peskine (1996), p.62.
} 


$$
N_{1}^{F}=\frac{\left(1-E^{2}\right) \bar{N}_{1}+E(1+E)\left(\bar{N}_{2}+\bar{N}_{3}\right)}{1-3 E^{2}-2 E^{3}} .
$$

Similarly to Section II, the networking cost function is $C\left(N_{1}\right)=c_{n} N_{1}+c_{\bar{N}} \bar{N}_{1}$ or, substituting the formula for $\bar{N}_{1}$ as obtained from (10a):

$$
\begin{aligned}
& C\left(N_{1}, \bar{N}_{2}, \bar{N}_{3}\right)=\left(c_{n}+c_{\bar{N}} \frac{1-3 E^{2}-2 E^{3}}{1-E^{2}}\right) N_{1}-c_{\bar{N}} \frac{E(1+E)}{1-E^{2}}\left(\bar{N}_{2}+\bar{N}_{3}\right) . \\
& \partial C\left(N_{1}, \bar{N}_{2}, \bar{N}_{3}\right) / \partial N_{1}=c_{n}+c_{\bar{N}} \frac{1-3 E^{2}-2 E^{3}}{1-E^{2}}=C_{F}^{\prime} .
\end{aligned}
$$

Equation (11c) shows that marginal networking costs ${ }^{9}$ are an indirect function of $E$ : an increase of the externality parameter will decrease the marginal costs of the network. The second and the third term of equation (11b) show that $\bar{N}_{2}$ and $\bar{N}_{3}$ are subsidies on unit 1's cost function; however, as the term $\left(E+E^{2}\right) /\left(1-E^{2}\right)$ varies directly with respect to $E$, a decrease of the externality parameter increases the networking costs, since it decreases the value of $\bar{N}_{2}$ and $\bar{N}_{3}$ as strategic substitutes or as subsidies on $\operatorname{costs}^{10}$. Hence, while in a 2nodes network we have focused only on the second effect, here we analyze the trade-off between the efficiency of direct links and the efficiency of the indirect ones.

The unit's minimization problem now takes into account the production constraint and the networking function, as defined by (11a). Solving the Lagrangian associated with the firm's minimization program, we obtain the same structure of input demand as in paragraph II.2. At the optimum, $N_{1}^{* F}$ is a negative function of the marginal networking costs:

$$
N_{1}^{* F}=\frac{\eta^{\prime}}{\left(C_{F}^{\prime}\right)^{\beta}}
$$

where:

$$
\eta^{\prime}=Y^{*}\left(\frac{1-\beta}{\beta} c_{L}\right)^{\beta}
$$

Equation (12a) implies that network marginal costs are the only relevant factor for differentiating telecommunications equipment choice under different network

\footnotetext{
${ }^{9}$ If we look at the matrix form (9), networking costs can be written as follows:

$C\left(N_{1}, \bar{N}_{1}\right)=\left(c_{n}+c_{\bar{N}} \frac{D}{\operatorname{cof} 1}\right) N_{1}-c_{\bar{N}} \frac{\operatorname{cof} 1}{\operatorname{cof} 2}\left(\bar{N}_{2}+\bar{N}_{3}\right)$

where $D$ is the determinant of $(I-\widetilde{E})^{-1}$ and $\operatorname{cof} i$, with $i=1,2$ is the co-factor of the row $i$-column $i$ factor of the same matrix. Therefore, the condition ensuring positive $N_{i}$ also ensures positive marginal networking costs.

${ }^{10}$ If we give up the hypothesis on isotropic externalities, evaluating the extent of $\bar{N}_{2}$ and $\bar{N}_{3}$ as strategic substitutes is more complicated. In fact, their degree of substitutability depends not only on the efficiency of direct contacts between unit 1 and units 2 or 3, but also from the two-way exchange between units 2 and 3 . In particular, the elasticity of substitution between $\bar{N}_{1}$ and $\bar{N}_{2}$ (measured as $\left|\partial \bar{N}_{1} / \partial \bar{N}_{2}\right|$ ) increases with greater benefit of the direct contact between unit 1 and unit 2 , but also on the efficiency of the two-ways exchanges between unit 2 and unit 3 and of the communications between unit 1 and unit 2 through unit 3 .
} 
configurations which the units may belong. Combining the network demand with the networking function, we obtain unit 1's reaction function under the full connectivity network structure, that is:

$$
\bar{N}_{1}^{F}=\frac{1}{1-E^{2}}\left[\left(1-2 E^{2}-3 E^{3}\right) N_{1}^{* F}-E(1+E)\left(\bar{N}_{2}+\bar{N}_{3}\right)\right]
$$

\subsubsection{Serial and partial connectivity}

To obtain the networking functions in the case of serial connectivity, we replace in the system of linear equations (9) the following matrix of externality parameters:

$$
\widetilde{E}_{S}=\left[\begin{array}{ccc}
0 & E & 0 \\
E & 0 & E \\
0 & E & 0
\end{array}\right] .
$$

Positive values of $N$ for each unit will exist when $E<0.7$.

Unit 1's networking function and costs in the serial connectivity case (henceforth denoted by $S$ ) are as follows (respectively):

$$
\begin{aligned}
& N_{1}^{S}=\frac{1-E^{2}}{1-2 E^{2}} \bar{N}_{1}+\frac{E}{1-2 E^{2}}\left(\bar{N}_{2}+E \bar{N}_{3}\right) \\
& C\left(N_{1}, \bar{N}_{2}, \bar{N}_{3}\right)=\left(c_{n}+c_{\bar{N}} \frac{1-2 E^{2}}{1-E^{2}}\right) N_{1}-c_{\bar{N}} \frac{E}{1-E^{2}}\left(\bar{N}_{2}+E \bar{N}_{3}\right) . \\
& \partial C\left(N_{1}, \bar{N}_{2}, \bar{N}_{3}\right) / \partial N_{1}=\left(c_{n}+c_{\bar{N}} \frac{1-2 E^{2}}{1-E^{2}}\right)=C_{S}^{\prime} .
\end{aligned}
$$

Even if firm 1 is not directly linked to unit 3, the existence of a link between units 2 and 3 allows unit 1 to benefit from $\bar{N}_{3}$ as a strategic substitute for $\bar{N}_{1}$. There is always a trade-off between the productivity and the network effect, but with a lesser extent than in the full connectivity configuration. On one side, less direct link implies lower marginal costs; on the other side, when there is only an indirect link unit 1 to unit 3 , it is clear that $\bar{N}_{3}$ is a weaker substitute than $\bar{N}_{2}$.

Combining the network demand with the networking function, we obtain unit 1 's networking function of the serial connectivity case $\left(\bar{N}_{1}^{S}\right)$ :

$$
\bar{N}_{1}^{S}=\frac{1}{1-E^{2}}\left[\left(1-2 E^{2}\right) N_{1}^{* S}-E\left(\bar{N}_{2}+E \bar{N}_{3}\right)\right]
$$

where

$$
N_{1}^{* S}=\frac{\eta^{\prime}}{\left(C^{\prime}\right)^{\beta}},
$$

and $\eta^{\prime}$ is defined by $(12 \mathrm{~b})$. In the serial connectivity configuration, units are asymmetric: units 2 and 3 are in a partial connectivity configuration that we will illustrate, taking unit 1 as an example. In the partial connectivity configuration (henceforth $P$ ), we solve the system of linear equation by using the following matrix of externality parameters: 


$$
\widetilde{E}_{P}=\left[\begin{array}{ccc}
0 & E & E \\
E & 0 & 0 \\
E & 0 & 0
\end{array}\right] .
$$

We consider values of $E<0.7$ for the usual positivity constraints. Unit 1's networking function and costs are as follows (respectively):

$$
\begin{aligned}
& N_{1}^{P}=\frac{1}{1-2 E^{2}} \bar{N}_{1}+\frac{E}{1-2 E^{2}}\left(\bar{N}_{2}+\bar{N}_{3}\right) . \\
& C\left(N_{1}, \bar{N}_{2}, \bar{N}_{3}\right)=\left(c_{n}+\left(1-2 E^{2}\right) c_{\bar{N}}\right) N_{1}-c_{\bar{N}} E\left(\bar{N}_{2}+\bar{N}_{3}\right) . \\
& \partial C\left(N_{1}, \bar{N}_{2}, \bar{N}_{3}\right) / \partial N_{1}=\left(c_{n}+\left(1-2 E^{2}\right) c_{\bar{N}}\right)=C^{\prime}{ }_{P} .
\end{aligned}
$$

Combining the network demand with the networking functions, we obtain the reaction function $\bar{N}_{1}^{P}$, as a function of $\bar{N}_{2}$ and $\bar{N}_{3}$ :

$$
\bar{N}_{1}^{P}=\left(1-2 E^{2}\right) N_{1}^{P}-E\left(\bar{N}_{2}+\bar{N}_{3}\right),
$$

where:

$$
N_{1}^{* P}=\frac{\eta^{\prime}}{\left(C^{\prime}{ }_{P}\right)^{\beta}},
$$

and $\eta^{\prime}$ is defined by (12b). $\bar{N}_{2}$ and $\bar{N}_{3}$ are still strategic substitutes for $\bar{N}_{1}$, but now, as the link is absent from unit 2 to unit 3, both the productivity and the network effect decrease with respect to the previous cases. However, the importance of $\bar{N}_{3}$ as a substitute (measured as $\left|\partial \bar{N}_{1}^{P} / \partial \bar{N}_{2}\right|$ ) is higher than in the serial connectivity configuration, as a direct link unit 1 to unit 3 does exist.

\subsection{Comparison of reaction functions and Nash equilibrium under the different network structures}

To understand the interdependence between network structure and telecommunications usage, it is useful to compare unit 1's reaction functions for different network configurations and the corresponding Nash equilibria, following the solution methodology of the simultaneous setting introduced in section 2 . We consider that, once each unit has chosen the telecommunication infrastructure, the usage of the network is determined by solving the system given by the networking functions that are treated as reaction functions.

The first step is to analyze the infrastructure choice. When the externality parameter is quite low, thus ensuring positive telecommunication infrastructure at the optimum in each networking configuration, the impact of the productivity effect on the demand of telecommunication infrastructure can easily be analyzed, obtaining the following result:

\section{Proposition 2}

When E<0.5, each node has the highest demand for input information in the full connectivity configuration. 
Proof: See the Appendix.

The global impact of the network and the productivity effect on the number of calls $\bar{N}$ is less clear-cut. On one hand, the increase in equipment demand associated with the full network connectivity case will increase $\bar{N}$, but, on the other hand, direct links offer the possibility of free-riding and benefiting from the telecommunications usage of the other nodes. Moreover, the higher the number of direct links, the higher the substitution possibilities. We then have to compare the relative magnitude of the strategic or free-riding network effect, linked to the interaction between firms, compared to the productivity effect, that could increase $N_{1}$ thus $\bar{N}_{1}$ via the reduction of marginal networking costs.

Two situations are representative of all the possible network configurations. In the first case, all units are symmetric (full connectivity), and in the second one, units are asymmetric, since unit 1 is in the partial connectivity configuration, while the others are in serial connectivity. The problem can easily be analyzed as a two-variable problem, where we compare $\bar{N}_{i}$ and the average usage of the other units belonging to the network $\left(\left(\bar{N}_{2}+\bar{N}_{3}\right) / 2=\bar{N}_{-i}\right)$. For the case of full connected networks, this is equivalent to imposing symmetry of the solutions; when comparing the case where unit 1 is in partial connectivity and units 2 and 3 in serial connectivity, the two-variable problem insulates the behavior of the symmetric units vis-à-vis the asymmetric one.

The system of reaction functions corresponding to the full connectivity case can be written as follows:

$$
\begin{aligned}
& \bar{N}_{i}^{F}=\frac{\eta^{\prime}\left(1-3 E^{2}-2 E^{3}\right)}{\left(C_{F}^{\prime}\right)^{\beta}\left(1-E^{2}\right)}-\frac{2 E}{(1-E)} \bar{N}_{-i}^{F} \\
& \bar{N}_{-i}^{F}=\frac{\eta^{\prime}\left(1-3 E^{2}-2 E^{3}\right)}{\left(C_{F}^{\prime}\right)^{\beta}(1+E)}-E \bar{N}^{F}{ }_{i} .
\end{aligned}
$$

The reaction functions system corresponding to partial connectivity for unit $i$ and to serial connectivity for the other units is:

$$
\begin{aligned}
& \bar{N}_{i}^{P}=\frac{\eta^{\prime}\left(1-2 E^{2}\right)}{\left(C_{P}^{\prime}\right)^{\beta}}-2 E \bar{N}^{S}{ }_{-i} \\
& \bar{N}_{-i}^{S}=\frac{\eta^{\prime}\left(1-2 E^{2}\right)}{\left(C_{S}^{\prime}\right)^{\beta}\left(1+E^{2}\right)}-\frac{2 E}{\left(1+E^{2}\right)} \bar{N}^{P}{ }_{i},
\end{aligned}
$$

where $C_{F}^{\prime}, C_{s}^{\prime}, C_{P}^{\prime}$ are defined respectively by equations (11c), (15c), (18c).

Analyzing the two systems of reaction functions, we obtain the following result on the equilibrium levels:

\section{Proposition 3}

When a stable, unique and positive Nash equilibrium exists for the both the full and the partial connectivity configurations $(E \in(0, \gamma)$ with $\gamma<0.5)$, we have 
$\bar{N}_{-i}^{* S}<\bar{N}_{1}^{* P}$ and $\bar{N}_{1}^{* F}=\bar{N}_{-i}^{* F}=\bar{N}^{* F}$. Moreover, the following inequalities hold: $\bar{N}_{1}^{* P}<\bar{N}_{1}^{* F}, \bar{N}_{-i}^{* S}<\bar{N}_{-i}^{* F}$.

Proof: See the Appendix.

With regards to the sensitivity of the optimal solutions to the externality parameter, it results that:

\section{Proposition 4}

In the case of full connectivity, an increase of the externality parameter has a negative effect on the demand of calls $\bar{N}_{i}^{F}$ and $\bar{N}_{-i}^{F}$. An increase (decrease) of the externality parameter $E$ increases (decreases) $\bar{N}_{1}^{* P}$ and decreases (increases) $\bar{N}_{-i}{ }^{* P}$.

Proof: See the Appendix.

We observe that the solutions associated with the full connected network are equal, because firms are totally symmetric. Most importantly, for the optimal set-up of an internal communication network, proposition 4 and 5 say that when units are identical, totally symmetric and externalities are isotropic and quite low, the full connected network ensures the higher number of contacts. It is also logical that, in the serial case, the average telecommunications usage of unit 2 and unit 3 is lower than that of the full connectivity case, since their number of direct link increases. More interesting is the result for unit 1 : her number of direct links is the same in the full and in the partial connectivity cases. Nevertheless, the existence of an indirect link between firms 2 and 3 stimulates further telecommunications usage. But when this indirect link exists, an increase of the externality parameter has the above-mentioned free-riding effect: unit 1 lowers her telecommunications usage, gaining from the higher network externality generated by the link unit 2 to unit 3.

Conversely, in the partial connectivity case, an increase in the externality parameter has a positive effect on unit 1's telecommunications usage, since there is higher benefit in communicating directly with the other units connected.

\section{Applications of the model and conclusions}

Our model has showed that when network externalities are embedded in the production function, the traditional idea that the higher the number of users, the higher the value of the network highly depends on the intensity of interaction among users.

The framework we propose can be useful in at least two potential applications: firstly, suggesting a solution to the problem of estimating telecommunications demand by multiunit firms, taking into account inter-firms calls, and the secondly, more broadly, looking at the relationship between firms' hierarchical organization and communication flows.

\subsection{Estimation of telecommunications demand by multi-unit firms}

With regards to the empirical implications, our paper shows that the architecture of internal communication networks affects overall firms' telecommunication demand. If data on 
inter-firms calls and their costs were available, these data would in fact be substituted in the cost function, calculating the share of national calls internal to the firms, and those going from the firm to outside users. If one possesses data on telecommunication equipment, outgoing and ingoing calls duration of intra-firm calls, the externality parameters could also be estimated.

However, it may be argued that because this data on intra-networks is almost impossible to obtain, our method does not really help in correctly estimating business telecommunications demand. For this reason, we stress that the information we need on internal communication networks could also be obtained in an indirect way that is inferring information on firms' internal communication network from plant localization. The first step to implement this method is to construct a symmetric distance matrix, that shows plants situated in national calls zone ${ }^{11}$ (as defined by the geographical criterion used for medium distance tariffs). The plants must be aggregated by each firm, and weighted by the plant size (in terms of number of employees), in order to obtain the percentage of national calls on total firms' inter-plant calls.

As an example, think of a firm $A$ with three plants $i, j, k$. Assume that our matrix indicates that communications between $i$ and $j$ and $j$ and $k$ are national calls. Under the hypothesis that the call distribution is isotropic, using the number of employees as a proxy for plant size (respectively $S_{i}, S_{j}, S_{k}$ ), the number of national connections among the total inter-plant (Aip) calls for firm $A$ can be defined as : $2 S_{i} S_{j}+2 S_{j} S_{k}=N_{\text {Aip }}$. The number of local and national connections can be normalized by dividing them by the total number of potential connections. Therefore, firm $A$ 's costs for national traffic are the weighted sum of inter-plant communications and total "other" communication at the national level. The shortcoming of this method is that it will overestimate the number of inter-firms calls: from our estimates (Creti-Bousquet-Wolkowitch, 2000), it was evident that observed traffic duration per employee does not increase when moving from medium to big firms. This may be due to the fact that, even in the presence of inter-firm network, in general only a fraction of plants is « on-net», otherwise the virtual solution would be too costly.

\subsection{Internal organization of firms}

Our results are easily extended to firms' organization: in section 2 we have already suggested the interpretation of the sequential model as the behavior of an M-form organization, where the marketing division could act as a leader in the communication decisions. We pointed out that the marketing division in the M-form would save the communication costs, inducing the production division to increase her calls ${ }^{12}$.

This simple example motivates the interest of further discussing our results in the context of the literature à la Radner $(1992,1993)$ focusing on communication and the hierarchical organization of administrations, clerical work, and production inside firms. Radner's analysis focuses on the structure and the size, that is, the number of nodes, of efficient hierarchies (defined as "ranked threes") that minimize delay in processing information. In order to focus on the importance of communication costs, we compare our

\footnotetext{
${ }^{11}$ We illustrate our ideas taking national traffic as an example, although the intuition remains valid for local and international calls.

${ }^{12} \mathrm{~A}$ more detailed analysis of communication flows, network externalities and oligopolistic competition with U-form and M-form organisations can be found in Creti, 2001.
} 
results with those of the Bolton-Dewatripont (henceforth B-D) model, which extends the Radner's basic model including costly communication.

In the B-D model, the internal organization of firms is seen as a communication network that is designed to minimize both the costs of processing new information and the costs of communicating this information among its agents. The B-D model analyzes two kinds of pyramidal networks: one form is the hierarchy in which each agent has an equal number of subordinates (the "regular pyramidal network"), and another one, in which each agent, except the last agent, has only one subordinate (the "conveyor belt"). The central idea of the paper is that is repeatedly processing information, agents become specialized, and the more they are specialized, the more communication is needed in order to coordinate the agents' activities and to aggregate the available information. The main result of the B-D work is that "an efficient network resembles a regular pyramid when it is efficient to have agents fully specialized in either processing or aggregating information. But an efficient network may also resemble an assembly line (the conveyor belt) when it is efficient for most agents to be involved in both processing or aggregating information [...] In most cases, the efficient network is similar to either or a combination of these two structures" (B-D, 1994).

A strong analogy exists between the conveyor belt and regular pyramidal network with the network configurations analyzed in section 3. To introduce the idea of hierarchical network in our model, we follow Radner's definition, that is, that each node can be ranked to represent a hierarchical level (or a production unit), which only communicates with the superior level. The serial connectivity structure corresponds to the regular pyramidal network, with unit 1 at the top level. The partial connectivity is the conveyor belt in terms of B-D model.

Before comparing some results obtained in section 3 with the main conclusions of the B-D model, we underline the main differences between the two approaches. The first difference is that, in our model, each node produces output combining labor, and processing information through interactions with other units belonging to a communication network. Therefore, processing information is not the only task of the agents. A second difference is that the B-D model deals with acyclic networks, where no agent receives, directly or indirectly, information from any of his direct or indirect superiors. On the contrary, we consider that each time a link between two nodes exists, a two-way communication is involved and that indirect links matter.

The third and most important difference is the modeling of communication costs. In the B-D model, communication costs are due to reading time. These costs involve a fixed cost of communication and a variable cost proportional to the item communicated. The positive returns to scale are due to the possibility of aggregating a given number of items processed. In our case, both network capacity and usage are included in the communication costs; moreover, those costs depend on contacts made by unit $i$ with directly and indirectly linked units, and on the externality parameter associated to these contacts. Therefore, the returns to information are specific to each network configuration.

Despite these differences, our model can easily deal with the same issues as those analyzed by B-D. In particular, we can find the optimal communication network, as the structure where production and networking costs are minimized. Imagine that a decisionmaker minimizes the sum of the production and networking costs of units $1,2,3$, and he has to choose between two structures, that is the conveyor belt (serial connectivity case) or the regular pyramidal network (partial connectivity). In our model, the trade-off between 
the two network configurations does not exist: minimizing the sum of the production and networking costs of each node, the regular pyramidal network and the conveyor belt are equally efficient. In fact, total networking costs are the same in the two structures: in each case, two firms are in the serial connectivity, and only one in the partial connectivity case. Network architecture is only differentiated at the unit level, where marginal costs of the network change, depending on direct and indirect links.

As empirical work (Bousquet-Creti-Wolkowicz, 2000; and Delmastro, 2004) suggest that a strong link between the firm's organization and the structure of the telecommunication network exist, we may find properties of pyramidal networks that are better suited to specific forms of corporate organization. For example, looking at networks that minimize the production and the networking marginal costs of the top-level hierarchy (node 1), under the hypothesis that all units simultaneously minimize their production and networking costs, we have the following result: the top-level hierarchy prefers the conveyor belt structure to the regular pyramidal network, with some restriction on the value of the externality parameter (see Proposition 4). This "decentralization effect" can also be obtained in the B-D model, allowing a decrease in communication costs. It is interesting to note that there is also some empirical evidence that the computerization of firms leads to smaller and flatter organizations, thus to greater decentralization (Brinjolfsson et al. 2005).

These are just a few examples showing that modeling network externalities in the production function may offer interesting criteria to analyze the relative efficiency of different inter-firm communication architectures. Indeed, our framework could be easily generalized to analyze properties of other kind of pyramidal networks, better suited to specific forms of corporate organization. In fact, we think that understanding how authority relationships interact with the incentives to communicate within a firm is a fascinating question, to which our model offers some stylized answers.

\section{$5 \quad$ References}

Artle R. and C. Averous (1973) "The Telephone System as a Public Good: Static and Dynamic Aspects," Bell Journal of Economics and Management Science, 4: 89-100.

Bolton P. and M. Dewatripont (1994) “The Firm as a Communication Network," Quarterly Journal of Economics, Vol. 109: 809-839.

Bousquet A., Creti A., and M. Wolkowicz (2000) "Analyse de la demande de telecommunications: une application sur données en coupe," Working Paper, France Télécom-Division Marketing n. 29.

Bousquet A. and M. Ivaldi (1997) "An Econometric Implementation," Information Economics and Policy, 9: 219-239.

Bousquet A. and D. Joram (1994) "Analyse du Trafic et de la Tarification du Service Colisée Numeris," mimeo, France Télécom-Cent. 
Brinjolfsson E. and M. Van Alstine (2005) "Global Village or Cyberbalkans: Modeling and Measuring the Integration of Electronic Communities," Management Science, 51: 851868.

Carter M. J. (1996) "Information and the Division of Labour: Implications for the Firm's Choice of Organisation," in M. Casson (ed.), The Theory of the Firm, Edward Elgar, Cheltenham, 267-79.

Creti A. (2001) "Firms' Organisation and Efficient Communication Networks," The Manchester Journal, 28: 85-102.

De Bondt, J. (1996) "R\&D and R\&D Spillovers: A Survey," International Journal of Industrial Organisation, 3: 30-56.

Curien N. and M. Gensollen (1989) Prévision de la Demande de Télécommunications. Eyrolles: Paris.

Delmastro, M. (2004) "The Determinants of the Management Hierarchy: Evidence from Italian Plants," International Journal of Industrial Organisation, vol.52 :53-80.

Dutta B. and S. Mutuswami (1997) "Stable Networks," Journal of Economic Theory, 76: 322-344.

Economides, N. (1991) "Compatibility and Market Structure," Working Paper, New York University, n. 321 Leonard Stern School of Business.

Gal-Or E. (1993) "Strategic Cost Allocation," The Journal of Industrial Economics, Vol 41: 387-402.

Griffin J. and B. Egan (1989) "Business Intercity Telecommunication Services," Review of Economics and Statistics, 30: 520-524.

Jackson M. and A. Wolinski (1996) "A Strategic Model of Social and Economic Networks," Journal of Economic Theory, 71: 44-74.

Katz, M. and C. Shapiro (1985) "Network Externalities, Competition and Compatibility," The American Economic Review, 75: 424-440.

Katz, M. and C. Shapiro (1986) "Technology Adoption in Presence of Network Externalities," Journal of Political Economy, 94: 822-841.

Liebowitz, S. and S. Margolis (1994) "Network Externality: An Uncommon Tragedy," Journal of Economic Perspectives, 8: 12-23.

Marinucci M. and Perez-Amaral T. (2005) Econometric Modeling of Business Telecommunications Demand using RETINA, mimeo, Universidad Complutense, Madrid.

Odlyzko A. (2000) "The Internet and Other Networks: Utilization Rates and their Implications," Information Economics and Policy, 12: 341-365. 
Pedrez-Amaral T., Alvarez-Gonzalez F., and B. M. Jimenez (1995) "Business Telephone Traffic Demand in Spain: 1980-1991, An Econometric Approach," Information Economic and Policy, 36: 60-75.

Radner R. (1992) "Hierarchy: The Economics of Managing," Journal of Economic Literature, Vol. 30: 1382-1415.

Radner R. (1993) "Information Processing in Firms and Returns to Scale," Annales d'Economie et Statistique, Vol.25/26: 265-298.

Rohlfs, J. (1974) “A Theory of Interdependent Demand for Communications Service," Bell Journal of Economics and Management Science, 5: 16-37.

Squire, L. (1973) "Some Aspects of Optimal Pricing for Telecommunications," Bell Journal of Economics and Management Science, 4: 515-525.

Taylor, L.D. (1994) Telecomunications Demand in Theory and Policy. Kluwer Academic Publishers: Dordrecht.

\section{$6 \quad$ Appendix}

\section{Proof of Proposition 1}

It is straightforward to note that the only differences between the firm 1's simultaneous and leader-follower (henceforth $l$ ) model's demand functions are the following terms of networking marginal costs:

$$
C^{\prime}\left(N_{1}\right)=c_{n}+c_{\bar{N}} \quad C^{\prime}\left(N_{1}^{l}\right)=c_{n}+c_{\bar{N}}\left(\frac{1}{1-\varepsilon_{21} \varepsilon_{12}}\right) .
$$

Under the assumptions on the externality parameters, we have $1-\varepsilon_{21} \varepsilon_{12}<1$, which means that $C^{\prime}\left(N_{1}\right)<C^{\prime}\left(N_{1}^{l}\right)$ or $N_{1}^{*}>N_{1}^{* l}$, since the demand of telecommunication services (ceteris paribus) is negatively related to networking marginal costs.

\section{Proof of Corollary 1}

Number of calls generated by the leader. At the leader-follower equilibrium, unit 1's volume of information is as follows:

$$
N_{1}^{l}=\bar{N}_{1}^{* l}+\varepsilon_{21} \bar{N}_{2}\left(\bar{N}_{1}^{* l}\right)=\bar{N}_{1}^{* l}+\varepsilon_{21}\left[N_{2}^{*}-\varepsilon_{12} \bar{N}_{1}^{* l}\right]=\bar{N}_{1}^{* l}\left(1-\varepsilon_{21} \varepsilon_{12}\right)+\varepsilon_{21} N_{2}^{*},
$$

where:

$$
N_{2}^{*}=Y_{2} *\left(\frac{1-\beta}{\beta} \frac{c_{L}}{c_{n}+c_{\bar{N}}}\right)^{\beta} .
$$

The term $1-\left(\varepsilon_{21} \varepsilon_{12}\right)$ being positive by assumption, a decrease of $N_{1}^{* l}$ implies a decrease of $\bar{N}_{1}^{l}$.

Number of calls generated by the follower (henceforth $f$ ). As for the equilibrium number of calls, we compare the following equations:

$$
\begin{aligned}
& \bar{N}_{2} *=\mathrm{N}_{2}^{*}-\varepsilon_{12} \bar{N}_{1}^{* l}, \\
& \bar{N}_{2}{ }^{f}=\mathrm{N}_{2}^{*}-\varepsilon_{12} \bar{N}_{1}^{*} .
\end{aligned}
$$


Since the first term of RHS is the same in both equations, and we know from the previous proof that $\bar{N}_{1}^{*}>\bar{N}_{1}^{*}$, the second term of RHS of equation (23a) will be lower than in (23b). We then conclude that $\bar{N}_{2}^{*}<\bar{N}_{2}^{*}$.

Technical rate of substitution between labor and communication services. In the case where network externalities are not present, the unit 1's technical rate of substitution between labor and communication equipment (that is, the ratio marginal productivity of $N$ over marginal productivity of $L$, henceforth $T R S$ ) is as follows:

$$
\text { TRS }=\frac{c_{n}}{c_{L}} .
$$

Taking into account network externalities and symmetric behavior, the TRS becomes:

$$
\operatorname{TRS}=\frac{\left(c_{n}+c_{\bar{N}}\right)}{c_{L}}=\mathrm{TRS}+\frac{c_{\bar{N}}}{c_{L}} .
$$

When unit 1 is the leader, the TRS will be the following:

$$
\operatorname{TRS}^{l}=\frac{\left(c_{n} B+c_{\bar{N}}\right)}{B c_{L}}=\mathrm{TRS}+\frac{c_{\bar{N}}}{c_{L} B} .
$$

From $B^{\prime}=1-\varepsilon_{21} \varepsilon_{12}<1$, it immediately follows: $T R S^{l}>T R S^{\prime}>T R S$.

\section{Proof of Proposition 2}

Demand for telecommunication infrastructure is a negative function of networking costs, ceteris paribus. Those networking costs only differ because of the intensity of the productivity effect:

$$
\begin{array}{ll}
C^{\prime}\left(N_{i}^{F}\right)=c_{n}+c_{\bar{N}} \frac{1-2 E^{2}-3 E^{3}}{1-E^{2}}, & \\
C^{\prime}\left(N_{i}^{S}\right)=c_{n}+c_{\bar{N}} \frac{1-2 E^{2}}{1-E^{2}}, & \\
C^{\prime}\left(N_{i}^{P}\right)=c_{n}+\left(1-2 E^{2}\right) c_{\bar{N}} . & i=1,2,3
\end{array}
$$

For $E<0.5$ (to obtain positive $N_{i}$ in the three network configurations)

$$
\frac{1-2 E^{2}-3 E^{3}}{1-2 E^{2}}<1-2 E^{2}<\frac{1-2 E^{2}}{1-E^{2}} .
$$

It immediately follows $N_{i}^{F}>N_{i}^{P}>N_{i}^{S}$.

\section{Proof of Proposition 3}

This proof requires some preliminary steps: in both the full connectivity and the partial/serial connectivity configurations, we find the conditions for stable and positive solutions, and we compare the Nash equilibrium of unit 1 with that of the other units (step 1 and 2). Each of these steps gives conditions on the value of the externality parameter, which have to be taken into account, when proving Proposition 4 (step 3).

\section{Step 1: Conditions on the full connectivity Nash equilibrium} CS: Stability 
A sufficient condition for the Nash equilibrium to be stable and unique ${ }^{13}$ in the full network connectivity configuration is that the externality parameter is sufficiently low $(E \in(0,0.5))$.

A sufficient condition for the Nash equilibrium to be stable is that the unit's 1 reaction function is steeper than the other firm's reaction function. This conditions means:

$$
\left|\frac{2 E}{1-E}\right|<\left|\frac{1}{E}\right| \Leftrightarrow 2 E^{2}+E-1<0 .
$$

Since $\mathrm{E}$ is always positive, this condition is satisfied with $E \in(0,0.5)$.

\section{CN: Positive equilibrium}

A necessary condition for the Nash-full connectivity equilibrium solutions to be positive is that the externality parameter is low $(E \in(0,0.5))$.

The necessary condition for the equilibrium to exist with positive $\bar{N}_{1}^{*}$ (where $F$ stands for full connectivity) is as follows:

$$
\frac{\eta^{\prime}\left(1-3 E^{2}-2 E^{3}\right)(1-E)}{\left(C_{F}^{\prime}\right)^{\beta}\left(1-E^{2}\right) 2 E}>\frac{\eta^{\prime}\left(1-3 E^{2}-2 E^{3}\right)}{\left(C_{F}^{\prime}\right)^{\beta}(1+E)^{2}} \Leftrightarrow E<1 .
$$

This condition is always satisfied by hypothesis.

Positive $\bar{N}_{-i}^{*} F$ will be obtained if:

$$
\frac{\eta^{\prime}\left(1-3 E^{2}-2 E^{3}\right)}{\left(C_{F}^{\prime}\right)^{\beta}(1-E)^{2}}<\frac{\eta^{\prime}\left(1-3 E^{2}-2 E^{3}\right)}{\left(C_{F}^{\prime}\right)^{\beta}(1+E)} \frac{1}{E} \Leftrightarrow E<0.5 .
$$

We then conclude that, when the externality parameter is lower than 0.5 , both $\bar{N}_{1}^{* F}$ and $\bar{N}_{-i}^{* F}$ are positive. This coincides with the condition to have positive networking functions.

Nash equilibrium solutions

When a stable and positive Nash equilibrium solution exist in the full connectivity case, the optimal solution for unit 1 is equal to that of the other firms $\left(\bar{N}_{1}^{*} F=\bar{N}_{-i}^{*}\right)$.

The system of the reaction functions gives the following Nash equilibrium solutions:

$$
\bar{N}_{1}^{* F}=\bar{N}_{-i}^{* F}=\frac{\eta^{\prime}(1-2 E)}{\left(C_{F}^{\prime}\right)^{\beta}} .
$$

\section{Step 2: Conditions on the partial/serial connectivity Nash equilibrium}

\section{CS: Stability}

A sufficient condition for the Nash equilibrium to be stable and unique in the partial network connectivity configuration is that the externality parameter is sufficiently low $(E \in(0,0.6))$.

The sufficient condition for the Nash equilibrium to be stable is:

$$
|2 E|<\left|\frac{1+E^{2}}{2 E}\right| \Leftrightarrow 3 E^{2}-1<0
$$

Since $\mathrm{E}$ is always positive, this condition is satisfied when $E \in(0,1 / \sqrt{3})$ or $E \in(0,0.6)$.

\section{CN: Positive equilibrium}

$\exists \gamma \in(0,0.5)$ such that a positive partial connectivity Nash equilibrium exists. The lower is the unit variable cost of network compared to the unit cost of capacity, the higher $\gamma$ will be.

\footnotetext{
${ }^{13}$ Since the reaction functions are linear, if a stable equilibrium exists, it is unique.
} 
The necessary condition for the equilibrium to exist with positive $\bar{N}_{1}^{* P}$ (where $P$ stands for partial connectivity) is as follows:

$$
\frac{\eta^{\prime}\left(1-2 E^{2}\right)}{2 E\left(C_{P}^{\prime}\right)^{\beta}}>\frac{\eta^{\prime}\left(1-2 E^{2}\right)}{\left(C_{S}^{\prime}\right)^{\beta}\left(1+E^{2}\right)} \Leftrightarrow\left(\frac{C_{S}^{\prime}}{C_{P}^{\prime}}\right)^{\beta}<\frac{2 E}{1+E^{2}} .
$$

Positive $\bar{N}_{-i}^{*}$ (where $S$ stands for serial connectivity) will be obtained if:

$$
\frac{\eta^{\prime}\left(1-2 E^{2}\right)}{\left(C_{P}^{\prime}\right)^{\beta}}>\frac{\eta^{\prime}\left(1-2 E^{2}\right)}{\left(C_{S}^{\prime}\right)^{\beta}\left(1+E^{2}\right)} \frac{\left(1+E^{2}\right)}{2 E} \Leftrightarrow\left(\frac{C_{S}^{\prime}}{C_{P}^{\prime}}\right)^{\beta}<\frac{1}{2 E} .
$$

We then have to find the conditions under which the following inequalities are satisfied:

$$
\frac{2 E}{1+E^{2}}<\left(\frac{C_{S}^{\prime}}{C_{P}^{\prime}}\right)^{\beta}<\frac{1}{2 E} \text {. }
$$

A qualitative study of the function $f_{1}=\left(C_{S}^{\prime} / C_{P}^{\prime}\right)^{\beta}$ is required in the interval $E \in(0,0.6)$, where the Nash solutions are stable. We know that $C_{S}^{\prime}>C_{P}^{\prime}$, and then $f_{1}>1$. That function depends positively on the unit variable $\operatorname{cost} c_{\bar{N}}$ and negatively on the unit cost of capacity $c_{n}$. Moreover, in the interval $E \in(0,0.5), f_{1}$ we have:

$$
\frac{\partial f_{1}}{\partial E}=\beta\left(f_{1}\right)^{\beta-1} c_{\bar{N}} \frac{2 E}{\left[c_{n}\left(1-E^{2}\right)+c_{\bar{N}}\left(1-E^{2}\right)\left(1-2 E^{2}\right)\right]^{2}}\left[\left(c_{n}+c_{\bar{N}}\right)\left(1-4 E^{2}\right)+2 E^{4}\left(c_{n}+2 c_{\bar{N}}\right)\right]
$$

The sign depends on the last term in the brackets. Since $\left(1-4 E^{2}\right)$ is positive in $E \in(0,0.5)$, and all the other terms are positive, we conclude that the derivative is poitive as well in that interval. Unfortunately, the sign of the derivative of $f_{1}$ with respect to $E$ in the interval $E \in(0.5,0.6)$ and the convexity/concavity with respect to $E$ are quite difficult to prove, without giving a numerical value to $c_{\bar{N}}$ and $c_{n}$. Numerical simulations show that the less $c_{n}$ increases with respect to $c_{\bar{N}}$, the more the function is convex.

The function $f_{2}=\frac{2 E}{1+E^{2}}$ is an increasing function of $E$, and it attains 1 when $E=1$. This means that the inequality $\frac{2 E}{1+E^{2}}<\left(\frac{C_{S}^{\prime}}{C_{P}^{\prime}}\right)^{\beta}$ is always verified in the interval $E \in(0,0.6)$.

The function $f_{3}=\frac{1}{2 E}$ is a decreasing function of $E$, and it attains 1 when $E=1 / 2$.

Comparing the plots of $f_{3}$ and $f_{1}$, we see that there exists a value of $\mathrm{E}, \gamma \in(0,0.5)$, so that the inequality is $\left(\frac{C_{S}^{\prime}}{C_{P}^{\prime}}\right)^{\beta}<\frac{1}{2 E}$ is satisfied (see Figure 2).

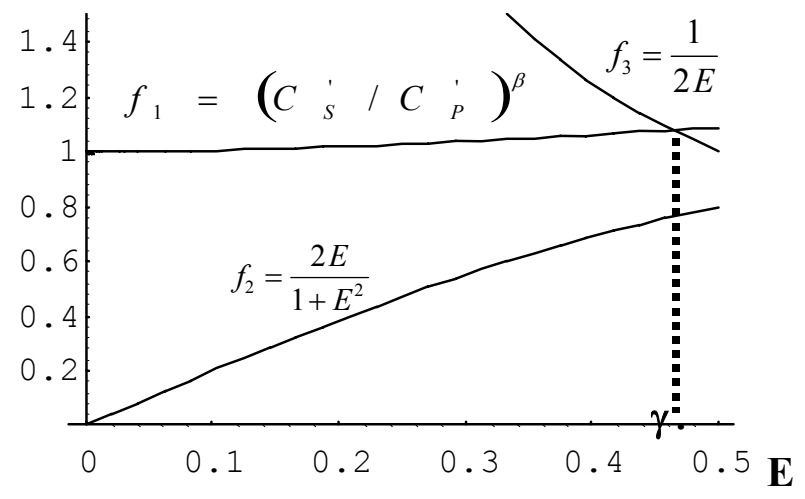


Figure 2 Comparison of $f_{1}=\left(C_{S}^{\prime} / C_{P}^{\prime}\right)^{\beta}$ with $f_{3}=\frac{1}{2 E}$ and $f_{2}=\frac{2 E}{1+E^{2}}$

Nash equilibrium solutions

When a stable and positive Nash equilibrium solution exists in the partial connectivity case, the optimal solution for unit 1 is higher than that of the other units $\left(\bar{N}_{1}^{* P}>\bar{N}_{-i}^{* S}\right)$.

The system of the reaction functions gives the following Nash equilibrium solutions:

$$
\begin{aligned}
& \bar{N}_{1}^{*_{P}}=\frac{\eta^{\prime}\left(2 E^{2}-1\right)}{\left(3 E^{2}-1\right)}\left[\frac{1}{\left(C_{P}^{\prime}\right)^{\beta}}-\frac{2 E}{\left(C_{S}^{\prime}\right)^{\beta}}+\frac{E^{2}}{\left(C_{P}^{\prime}\right)^{\beta}}\right], \\
& \bar{N}_{-i}^{*_{S}}=\frac{\eta^{\prime}\left(2 E^{2}-1\right)}{\left(3 E^{2}-1\right)}\left[\frac{1}{\left(C_{S}^{\prime}\right)^{\beta}}-\frac{2 E}{\left(C_{P}^{\prime}\right)^{\beta}}\right] .
\end{aligned}
$$

Their difference is then:

$$
\bar{N}_{1}^{* P}-\bar{N}_{-i}^{*}=\frac{\eta\left(2 E^{2}-1\right)}{\left(3 E^{2}-1\right)}\left\{\frac{E^{2}}{\left(C_{P}\right)^{\beta}}+2 E\left[\frac{1}{\left(C_{P}\right)^{\beta}}-\frac{1}{\left(C_{S}\right)^{\beta}}\right]+\left[\frac{1}{\left(C_{P}\right)^{\beta}}-\frac{1}{\left(C_{S}\right)^{\beta}}\right]\right\} \text {. }
$$

We know that for the stability condition, $\left(3 E^{2}-1\right)<0$, and then also $\left(2 E^{2}-1\right)<0$. We have only to study the sign of the last term on the right hand side of equation (31). Simple manipulations show that this term is positive if:

$$
\frac{(E+1)^{2}}{(2 E+1)}>\left(\frac{C_{P}^{\prime}}{C_{S}^{\prime}}\right)^{\beta} \text {. }
$$

This inequality is always verified, since $(E+1)^{2} /(2 E+1)$ is an increasing function of $E$ and always higher than one, while $\left(C_{P}^{\prime} / C_{S}^{\prime}\right)^{\beta}$ is a decreasing function of $\mathrm{E}$, and always less than 1. We conclude that the sign of the difference $\bar{N}_{1}^{* P}-\bar{N}_{-i}^{* S}$ is positive, and therefore $\bar{N}_{1}^{* P}>\bar{N}_{-i}^{* S}$.

\section{Step 3: Comparison of the full versus partial/serial solutions}

The full and partial connectivity Nash equilibrium for unit 1 can be factorized as follows:

$$
\begin{aligned}
& \bar{N}_{1}^{* F}=\frac{\eta^{\prime}(1-2 E)}{\left(C_{F}^{\prime}\right)^{\beta}}, \\
& \bar{N}_{1}^{* P}=\frac{\eta^{\prime}\left(2 E^{2}-1\right)}{\left(C_{P} C_{S}^{\prime}\right)^{\beta}\left(3 E^{2}-1\right)}\left[\left(C_{S}^{\prime}\right)^{\beta}-2 E\left(C_{P}\right)^{\beta}+E^{2}\left(C_{S}^{\prime}\right)^{\beta}\right]
\end{aligned}
$$

Their difference is positive if:

$$
\left(\frac{C_{P}^{\prime} C_{S}^{\prime}}{C_{F}^{\prime}}\right)^{\beta}\left[\frac{1}{\left(C_{S}^{\prime}\right)^{\beta}-2 E\left(C_{P}^{\prime}\right)^{\beta}+E^{2}\left(C_{S}^{\prime}\right)^{\beta}}\right]>\frac{\left(2 E^{2}-1\right)}{\left(3 E^{2}-1\right)(1-2 E)} .
$$

It is easy to show that when $E \in(0,0.5)$, the right hand side of inequality (33) is positive. The first term of the left hand side is positive by hypothesis; as regards the second term, we have:

$$
\left(C_{S}^{\prime}\right)^{\beta}-2 E\left(C_{P}^{\prime}\right)^{\beta}+E^{2}\left(C_{S}^{\prime}\right)^{\beta}>0 \Leftrightarrow \frac{2 E}{1+E^{2}}<\left(\frac{C_{S}^{\prime}}{C_{P}^{\prime}}\right)^{\beta} .
$$


This is exactly the condition that has to be satisfied in order to have positive $\bar{N}_{-i}^{* S}$ solutions. The difficulty now arising is that we do not have the exact value of the parameter $\gamma$ Unfortunately, this value depends on the shape of $f_{1}=\left(C_{S}^{\prime} / C_{P}^{\prime}\right)^{\beta}$, and cannot be calculated without giving numerical values to the unit cost of capacity and to the unit variable cost. Our numerical simulations showed that, under a reasonable hypothesis on the value of unit variable costs and unit cost of capacity of the network, $f_{1}$ is slightly higher than 1 at least in the interval $E<0.5$, and that it increases very slowly. This means that the point at which $f_{1}$ and $f_{3}=1 / 2 E$ meet is very close to 0.5 (see Graph 1), as required to prove that the sign of the term analyzed is positive. We then conclude that the last inequality is always verified in the interval $(0, \gamma)$ where $\gamma$ is very close to 0.5 , and then $\bar{N}_{1}^{*} P \bar{N}_{1}^{*} F$. Moreover, we know from the previous steps that $\bar{N}_{-i}^{* S}<\bar{N}_{1}^{* P}$ and $\bar{N}_{1}^{* F}=\bar{N}_{-i}^{* F}$. Once it is shown that $\bar{N}_{1}^{* P}<\bar{N}_{1}^{* F}$, we easily have $\bar{N}_{-i}^{* S}<\bar{N}_{1}^{* P}<\bar{N}_{1}^{* F}=\bar{N}_{-i}^{* F}$, and then $\bar{N}_{-i}^{* S}<\bar{N}_{-i}^{* F}$.

\section{Proof of Proposition 4}

The derivative of $\bar{N}_{1}^{*}$ with respect to $\mathrm{E}$ is:

$$
\frac{\partial \bar{N}_{1}^{*} F}{\partial E}=\eta^{\prime}\left\{\left[\frac{-2}{\left(C_{F}^{\prime}\right)^{\beta}}\right]+\left[(1-2 E) \frac{\partial\left(C_{F}^{\prime}\right)^{-\beta}}{\partial E}\right]\right\} \text {. }
$$

It is easy to show that this derivative is negative if:

$$
\eta^{\prime} \beta\left(C_{F}^{\prime}\right)^{2 \beta-1} \frac{E}{\left(1-E^{2}\right)^{2}}\left(E^{2}-3 E-2\right)<1 .
$$

This inequality is always verified, since in $E \in(0, \gamma)$ with $\gamma<0.5$, the term $\left(E^{2}-3 E-2\right)$ is negative, and all the other terms are positive. For the partial/serial case, for computational convenience, we study $\partial \bar{N}_{-i}^{* S} / \partial E$. Remembering that:

and

$$
\bar{N}_{-i}^{*}=\frac{\eta^{\prime}\left(2 E^{2}-1\right)}{\left(3 E^{2}-1\right)}\left[\frac{1}{\left(C_{S}^{\prime}\right)^{\beta}}-\frac{2 E}{\left(C_{P}^{\prime}\right)^{\beta}}\right],
$$

$$
\begin{aligned}
& \frac{\left(2 E^{2}-1\right)}{\left(3 E^{2}-1\right)}>0 \text { when } \mathrm{E}<\gamma<0.5, \\
& \frac{\partial}{\partial E}\left[\frac{2 E^{2}-1}{3 E^{2}-1}\right]>0 \quad \forall \mathrm{E} .
\end{aligned}
$$

We study the sign of the derivative of the terms in square brackets. We first prove two preliminary results:

Result 1:

$$
\frac{\partial\left(C_{S}^{\prime}\right)^{-\beta}}{\partial E}<\frac{\partial\left(C_{P}^{\prime}\right)^{-\beta}}{\partial E}<2 \frac{\partial\left(C_{P}^{\prime}\right)^{-\beta}}{\partial E}
$$

Proof

Calculating the derivatives $\partial\left(C_{P}^{\prime}\right)^{-\beta} / \partial E$ and $\partial\left(C_{S}^{\prime}\right)^{-\beta} / \partial E$ we have:

$$
\frac{4 E \beta c_{\bar{N}}}{\left(C_{P}^{\prime}\right)^{\beta+1}}>\frac{2 E \beta c_{\bar{N}}}{\left(C_{S}^{\prime}\right)^{\beta+1}\left(1-E^{2}\right)^{2}} \Leftrightarrow\left(\frac{C_{P}^{\prime}}{C_{S}^{\prime}}\right)^{\beta+1}<2\left(1-E^{2}\right)^{2},
$$

since: 


$$
2\left(1-E^{2}\right)^{2}>1>\left(\frac{C_{P}^{\prime}}{C_{S}^{\prime}}\right)^{\beta+1} .
$$

The inequality always holds in $E<0.5$, and then also in the interval of interest $(E<0.33)$. Note that the right hand side of Result 1 is always true, since the derivative $\partial\left(C_{P}^{\prime}\right)^{-\beta} / \partial E$ is positive.

Result 2:

$$
2 \frac{\partial\left(C_{P}^{\prime}\right)^{-\rho}}{\partial E}<\frac{\partial\left[E\left(C_{P}^{\prime}\right)^{-\rho}\right]}{\partial E}
$$

Proof

The above inequality can be written as:

$$
2 \frac{\partial\left(C_{P}^{\prime}\right)^{-\beta}}{\partial E}<E \frac{\partial\left(C_{P}^{\prime}\right)^{-\beta}}{\partial E}+\frac{1}{\left(C_{P}^{\prime}\right)^{\beta}} .
$$

We then have:

$$
(2-E) \frac{\partial\left(C_{P}^{\prime}\right)^{-\beta}}{\partial E}<\frac{1}{\left(C_{P}^{\prime}\right)^{\beta}} \Leftrightarrow c_{\bar{N}}\left[2 E^{2}(1-2 \beta)+8 \beta E-1\right]>c_{n} .
$$

This is true because:

$$
2 E^{2}(1-2 \beta)+8 \beta E-1>1
$$

and $c_{\bar{N}}>c_{n}$ by hypothesis.

We can now prove Proposition 3. Combining (36) and (37), we have:

$$
2 \frac{\partial\left[E\left(C_{P}^{\prime}\right)^{-\beta}\right]}{\partial E}>\frac{\partial\left[E\left(C_{P}^{\prime}\right)^{-\beta}\right]}{\partial E}>2 \frac{\partial\left(C_{P}^{\prime}\right)^{-\beta}}{\partial E}>\frac{\partial\left(C_{S}^{\prime}\right)^{-\beta}}{\partial E}
$$

then

$$
2 \frac{\partial\left[E\left(C_{P}^{\prime}\right)^{-\beta}\right]}{\partial E}>\frac{\partial\left(C_{S}^{\prime}\right)^{-\beta}}{\partial E} \text {. }
$$

It means that:

$$
\frac{\partial}{\partial E}\left[\frac{1}{\left(C_{S}^{\prime}\right)^{\beta}}-\frac{2 E}{\left(C_{P}^{\prime}\right)^{\beta}}\right]<0,
$$

or $\quad \overline{D N}_{-i}^{*} / \partial E<0$.

Similar arguments apply to show that $\partial \bar{N}_{1}^{* P} / \partial E>0$. 